\title{
Das Problem des religiösen Akosmismus in der Kierkegaard-Rezeption von Karl Jaspers
}

\begin{abstract}
Karl Jaspers' reception of Kierkegaard has been characterized by some commentators as a largely uncritical appropriation. In my paper I argue that this view is not entirely adequate since the former's attitude to the Danish thinker is by no means purely affirmative. By contrast, it seems to me that the punctum saliens of his criticism is the idea of a religiously motivated negation of the world (religiöse Weltverneinung), which certainly dominates the later thought of Kierkegaard. In order to justify my claim both Jaspers' existential concept of the world and that of a world negation will be dealt with. In the main part of the article I will try to reconstruct, from a historical point of view (a) the shaping and development of Jaspers' increasingly sharp criticism of Kierkegaard's “acosmic” view of Christianity and (b) his late self-distancing from the Danish thinker. In my concluding remarks I will point out that although his criticism is in principal not unfounded, it is nevertheless one-sided and reductionistic, since it is based mainly on Kierkegaard's polemical writings from the late period of his Kirchenkampf and as such ignores or at least underestimates some important aspects of Kierkegaard's early thought.
\end{abstract}

Bekanntlich wurde Kierkegaards Denken in der deutschen akademischen Philosophie vor dem ersten Weltkrieg bzw. vor der Veröffentlichung seiner Gesammelten Werke $^{1}$ beinahe völlig ignoriert. ${ }^{2}$ Es ist vor allem das Verdienst des Psychologen

Die vorliegende Studie wurde im Rahmen des Forschungsstipendiums Bolyai János der Ungarischen Akademie der Wissenschaften durchgeführt.

1 Søren Kierkegaard, Gesammelte Werke, Bde. 1-12, übers. und hg. von Hermann Gottsched und Christoph Schrempf, Jena 1909-22.

2 Allerdings bedeutet dies keineswegs, dass Kierkegaard in Deutschland vor dem Ende des 19. Jahrhunderts völlig unbekannt gewesen wäre: „Paul Tillich’s claim, that Kierkegaard remained ,völlig unbekannt' in Germany up until the late nineteenth century calls for correction“. Heiko Schulz, „Germany and Austria: A Modest Head Start: The German Reception of Kierkegaard“, in Kierkegaard's International Reception, Tome I, Northern and Western Europe, hg. von Jon Stewart, Aldershot 2009 (Kierkegaard Research: Sources, Reception and Resources, Bd. 8), S. 321.

István Czakó, Institute of Philosophy, Pázmány Péter Catholic University, 1 Egyetem u., 2087 Piliscsaba, Hungary, stephanus.czako@gmail.com 
und Philosophen Karl Jaspers (1883-1969), dass sich diese Situation mit der Zeit wesentlich verändert hat. Zum ersten Mal stieß jener um 1914 auf Kierkegaard und wurde sofort zum eifrigen Leser des Dänen. ${ }^{3}$ Kierkegaard taucht in seinen Universitätsvorlesungen regelmäßig auf und er widmete ihm ein ganzes Kapitel in seinem Frühwerk Psychologie der Weltanschauungen. ${ }^{4}$ Jaspers hielt Kierkegaard zusammen mit Friedrich Nietzsche für den wichtigsten Denker der post-Kantischen Zeit. ${ }^{5}$ Er charakterisiert das Wesen des (von ihm vorbehaltlos übernommenen) denkerischen Programms Kierkegaards mit dem folgenden Satz aus der Nachschrift: die „Urschrift der individuellen, humanen Existenzverhältnisse, das Alte, Bekannte und von den Vätern überlieferte noch einmal, womöglich auf eine innerliche Weise, zu lesen."6 Es ist für Jaspers darüber hinaus wesentlich, Kierkegaard nicht als einen Denker unter Anderen zu verstehen, sondern in ihm eine Ausnahme, einen Propheten der indirekten Mitteilung und der Redlichkeit, einen Sturmvogel ${ }^{7}$ des 19. Jahrhunderts zu sehen, der die kommende Katastrophe anzeigt - ohne eine beruhigende, positive Lehre darzulegen. Der dänische Denker repräsentiert für ihn keinen besonderen Standpunkt, sondern eher eine Denkart, die eben deshalb nie überwunden werden kann. Das ganze Kierkegaardsche Denken kulminiert für Jaspers in der Enthüllung der Existenzmöglichkeiten des existierenden Individuums in der Modernität. Daher ist es bestimmt kein Zufall, dass sein eigenes denkerisches Werk jenem Kierkegaards so nahe steht, dass man es Michael Theunissen zufolge - „als einen einzigen Kommentar zu Kierkegaard lesen“8 kann. Entsprechend maliziös bemerkt Jean-Paul Sartre, „Jaspers beschränke sich darauf, Kierkegaard zu kommentieren und habe bloß insofern

3 Ludwig Edelstein, „Erich Frank’s Work: An Appreciation“, in Erich Frank, Wissen Glauben Wollen: Gesammelte Aufsätze zur Philosophiegeschichte und Existentialphilosophie, hg. von Ludwig Edelstein, Zürich-Stuttgart 1955, S. 419.

4 Karl Jaspers, Psychologie der Weltanschauungen, Berlin 1919, S. 370-381.

5 „Während alle Philosophen nach Hegel ihnen [d.h. Kierkegaard und Nietzsche] gegenüber immer mehr zurücktreten, stehen sie als die eigentlich großen Denker ihres Zeitalters heute im Grunde schon unbezweifelt da: ihre Wirkung wie die Gegnerschaft gegen sie beweisen es." Karl Jaspers, Vernunft und Existenz, Groningen-Batavia 1935, S. 5.

6 Karl Jaspers, „Kierkegaard“, in ders., Aneignung und Polemik. Gesammelte Reden und Aufsätze zur Geschichte der Philosophie, München 1968, S. 297. Vgl. SKS 7, 573 / AUN2, 344.

7 Jaspers, „Kierkegaard“, in ders., Aneignung und Polemik, S. 309. Jaspers’ Text spielt an auf die folgende Passage bei Kierkegaard: „Es gibt einen Vogel, der Regenvogel genannt wird, und so bin ich, wenn in der Generation sich ein Unwetter zusammenzuziehen beginnt, dann erscheinen die Arten von Individualitäten, wie ich es bin.“ SKS 18, 291, JJ:391 / DSKE 2, 281.

8 Materialien zur Philosophie Søren Kierkegaards, hg. von Michael Theunissen und Wilfried Greve, Frankfurt a. M. 1979, S. 62. 
Anspruch auf Originalität, als er bestimmte Themen des Dänen herausarbeite und andere in den Hintergrund dränge“9.

Umso auffälliger ist deshalb, dass, obwohl Jaspers von Kierkegaards Existenzauffassung tief beeindruckt war und sogar den Existenzbegriff von ihm übernommen hat, ${ }^{10}$ seine Kierkegaard-Rezeption doch von einer permanenten Ambivalenz durchdrungen ist. Jaspers selbst betont, dass er kein „Anhänger“ Kierkegaards wurde, er war sogar einer der schärfsten Kritiker seiner Christentumsauffassung: „Ich blieb nicht nur unberührt von seinem Christentum, sondern spürte in seinen negativen Entschlüssen ... das Gegenteil von allem, was ich liebte und wollte, zu tun bereit und nicht bereit war. Seine Auffassung des christlichen Glaubens durch seine Religiosität B schien mir ebenso wie jene praktische Negativität das Ende des geschichtlichen Christentums und auch das Ende jeden philosophischen Lebens. "11 Aus dieser Aversion folgt bei Jaspers nicht nur eine negative Einstellung, sondern auch ein bereits in der Psychologie der Weltanschauungen eingeführtes, konsequent angewandtes methodisches Prinzip, und zwar jenes der systematischen Ausblendung aller christlichen Inhalte in seiner Kierkegaard-Rezeption. ${ }^{12}$ Diese selektive Methodologie führte praktisch dazu, dass Jaspers einige Teile des Kierkegaardschen Corpus (z. B. die Erbaulichen und die Christlichen Reden) einfach ignoriert. ${ }^{13}$ Es lässt sich deshalb zweifellos feststellen, dass für seine Kierkegaard-Rezeption - gegen den herkömmlichen Vorwurf einer kritiklosen Aneignung - eben eine kritische Haltung und eine konstitutive Ambivalenz charakteristisch ist. In den nachfolgenden Überlegungen versuche ich das Problem, das meines Erachtens den hauptsächlichen theoretischen Grund für die immanente Ambivalenz dieses rezeptiven Verhältnisses bildet, zu erläutern: Zunächst rekonstruiere ich den Begriff des religiösen Akos-

9 Ibid. Vgl. Jean-Paul Sarte, Critique de la raison dialectique, Bd. I., Paris 1960, S. 21.

10 „Kierkegaard verdanke ich den Begriff der 'Existenz', der mir seit 1916 maßgebend wurde, um das zu fassen, worum ich mich bis dahin in Unruhe bemüht hatte." Karl Jaspers, Philosophische Autobiographie, Erweiterte Neuausgabe, München 1977, S. 125.

11 Karl Jaspers, „Nachwort zu meiner Philosophie 1955“, in ders., Philosophie, Bd. I.: Philosophische Weltorientierung, Berlin-Heidelberg-New York 1973, S. XX.

12 Jaspers, Psychologie der Weltanschauungen, S. 370.

13 Siehe dazu Ehrlichs erhellende Darlegung von Jaspers' Kierkegaard-Lektüre: „Although the first part of The Sickness unto Death was of substantive importance to Jaspers' thinking, he never referred to the second part. Similarly, he placed the greatest value on Kierkegaard's philosophical pseudonyms, especially Climacus, and on his Journals, but never on the Edifying and Christian Discourses. Of the Christian writings Jaspers valued only the Attack upon Christendom.“ Leonard H. Ehrlich, „Editorial note to ,Reading Kierkegaard 1: Becoming Manifest““, in Karl Jaspers, Philosophy of History and History of Philosophy, hg. von Joseph W. Koterski und Raymond J. Langley, Amherst 2003, S. 245. 
mismus, der den Hintergrund der Jaspersschen Kritik an Kierkegaard bildet; sodann lege ich Jaspers’ Haupteinwände gegen Kierkegaards religiösen Weltverneinung dar; schließlich richte ich die Aufmerksamkeit auf einige problematische Aspekte von Jaspers’ Kritik an Kierkegaard.

\section{Der Begriff des religiösen Akosmismus}

\section{A Die Bestimmung des Akosmismus}

Gemäß Hans-Walter Schütte hat sich der Begriff „Akosmismus“ „in keiner eigenen philosophischen Theorie entfaltet; ihm kommt die im ganzen begrenzte Funktion $\mathrm{zu}$, eine unterschiedlichen Deutungen ausgesetzte Systembildung vor dem Vorwurf des Atheismus zu bewahren." ${ }^{14}$ So kommt der Ausdruck im Kontext des Jenaer Atheismusstreites in Fichtes „Gerichtlicher Verantwortungsschrift“ vor, in der Fichte seinen Kritiker bittet: „[D]enke er auf eine neue Bestimmung, nenne er mich etwa einen Akosmisten, nur nenne er mich nicht einen Atheisten. " ${ }^{15} \mathrm{Zu}$ nächst hatte der Leipziger Philosoph Ernst Platner behauptet, dass Spinoza eigentlich nicht die Existenz der Gottheit, sondern die Existenz der Welt leugne. Diese Konzeption kommt dann in Hegels Spinoza-Deutung völlig zum Durchbruch: „Bei Spinoza ist das Absolute die Substanz, und dem Endlichen wird kein Sein zugesprochen; es ist also Monotheismus, Akosmismus. So sehr ist nur Gott, dass gar keine Welt ist; das Endliche hat darin keine wahrhafte Wirklichkeit. " ${ }^{16}$ Es ist auffällig, dass diesem Begriff in Hegels Religionsphilosophie noch eine weitere, nämlich den gemeinen, besonders durch F.A.G. Tholuck erhobenen Vorwurf des Pantheismus neutralisierende Funktion zukommt, mit dem das System bereits zu Hegels Lebzeiten in Verbindung gebracht wurde. ${ }^{17}$

14 Hans-Walter Schütte, „Akosmismus“, in Historisches Wörterbuch der Philosophie, Bd. 1, hg. von Joachim Ritter et al., Basel-Stuttgart 1971, Sp. 128. Im Folgenden stütze ich mich größtenteils auf diesen Artikel.

15 Johann Gottlieb Fichte, „J. G. Fichte‘s als Verfassers des ersten angeklagten Aufsatzes und Mitherausgebers des phil. Journals Verantwortungsschrift“, in Fichtes Werke, hg. von Immanuel Hermann Fichte, Bd. 5.: Zur Religionsphilosophie, Berlin 1971, S. 269.

16 Georg Wilhelm Friedrich Hegel, Vorlesungen über die Philosophie der Religion, Teil 1: Einleitung in die Philosophie der Religion. Der Begriff der Religion, hg. von Walter Jaeschke, Hamburg 1993 (Philosophische Bibliothek, Bd. 459), S. 322.

17 Siehe dazu: Jon Stewart, „Hegel's Philosophy of Religion and the Question of ,Right' and ,Left' Hegelianism“, in Politics, Religion, and Art: Hegelian Debates, hg. von Douglas Moggach, Evanston, Illinois 2011, S. 76. 
Nicht zuletzt soll noch der im 20. Jahrhundert von Max Weber ausgebildete Begriff des Liebesakosmismus kurz erwähnt werden. ${ }^{18}$ Dieser soziologische Begriff bezeichnet ein gegenstandsloses Liebesgefühl im Kontext einer mystischkontemplativen Religiosität. Angesichts der Tatsache, dass Jaspers in Heidelberg eine intensive Arbeitsbeziehung mit Weber pflegte, liegt es nahe anzunehmen, dass ersterer in seiner Weber-Lektüre auch auf diesen religionssoziologischen Schüsselbegriff gestoßen ist. Um die eigentliche Relevanz der Akosmismus-Problematik in Jaspers' Denken aufzeigen zu können, soll im Folgenden sein Weltbegriff aus der Perspektive seines Hauptwerkes Philosophie (1931) und seiner Basler Vorlesungen Der philosophische Glaube (1947) in groben Zügen rekonstruiert werden.

\section{B Jaspers' existenzphilosophischer Weltbegriff}

Im ersten Teil seines Hauptwerkes arbeitet Jaspers den existenzphilosophischen Begriff der Welt heraus. Seine Konzeption ist metaphysisch weniger radikal als Heideggers fundamentalontologisch konzipierter, existentialanalytischer Begriff des Daseins als „In-der-Welt-Sein, “19 denn Jaspers hebt die Subjekt-Objekt Dichotomie nicht auf, betont aber doch ausdrücklich die Untrennbarkeit von Ich und Nicht-Ich: Die Einheit der Welt wird nicht vom subjektiven Dasein und auch nicht von der objektiven Wirklichkeit an sich gebildet, sondern beide Momente durchdringen sich wechselseitig. Im Abschnitt „Welt und Transzendenz“20 unterscheidet Jaspers zwischen zwei Gruppen von Weltbegriffen, je nachdem ob Welt an sich ohne ein Anderes, oder ob sie als Erscheinung in Bezug auf Existenz und Transzendenz gedacht wird:

\footnotetext{
Von reiner Weltorientierung her gesehen wird die Welt das dauernd Bestehende. Sie ist ohne Anfang und Ende, ohne Wandel, nur in ihr wandelt sich alles und hat Anfang und Ende...Ob ich diese Welt räumlich und mathematisch als das astronomische Weltall denke, oder zeitlich und dynamisch als die Natur,...immer habe ich ein Umschließendes, endlos Dauerndes im Gedanken, das als Universum selbstgenügsam in sich ruht in seinem gleichgültigen Dasein, und über das hinaus gar nicht gefragt werden kann.

Vom Transzendieren her gesehen aber ist diese Welt nur Dasein, das nicht aus sich ist, sondern Erscheinung. Der Mensch, sofern er nicht bloß Teil der Welt, sondern frei er selbst
}

18 Vgl. Max Weber, „Über einige Kategorien der verstehenden Soziologie“, in Logos 4, 1913, S. 253-294. Ich bedanke mich bei meinem Kollegen Zoltán Hidas, dass er meine Aufmerksamkeit auf diesen Problemzusammenhang gelenkt hat.

19 Vgl. Martin Heidegger, Sein und Zeit, 18. Aufl., Tübingen 2001, S. 52-62.

20 Jaspers, Philosophie, Bd. I, S. 81-84. 
sein kann, ist mögliche Existenz. Ihm als Bewusstsein überhaupt zeigt sich in der Weltorientierung die Welt als Welt, ihm als möglicher Existenz wird in der Welt Transzendenz offen. Für den Menschen als mögliche Existenz verliert die Welt ihre Gleichgültigkeit...Für ihn...ist die Welt das, worin und wodurch er sich mit anderer Existenz auf Transzendenz bezieht. ${ }^{21}$

Später in den Vorlesungen über den Philosophischen Glauben wird unter den philosophischen Glaubensinhalten folgende These formuliert: „Die Realität der Welt hat ein verschwindendes Dasein zwischen Gott und Existenz.“22 Durch diese Formel wird vor allem die Phänomenalität der Welt festgestellt, eine allgemeine Grundeinsicht in allen Formen der Transzendentalphilosophie. Die Welt wird in ihrer Totalität nicht zum Gegenstand für uns. Gott ist eine absolute Transzendenz in seinem Verhältnis zur Welt, er ist deus absconditus, zugleich ist er aber auch unverfügbar nah in der absoluten Geschichtlichkeit einer je einmaligen Situation. Die Welt ist für Jaspers nicht an sich, sondern in ihr vollzieht sich in bleibender Vieldeutigkeit die Sprache Gottes, jedoch nur in Form von Chiffren. Es gibt „kein direktes Wissen von Gott und der Existenz. Welterforschung ist der einzige Weg unseres Erkennens, Weltverwirklichung der einzige Weg existentieller Verwirklichung. In der Weltlosigkeit verlieren wir zugleich uns selbst.“23

\section{Der Begriff der religiösen Weltverneinung}

Der Begriff der religiösen Weltverneinung wird im Band „Existenzerhellung“ der Philosophie unter dem Titel „Unbedingte Handlungen“ systematisch ausgearbeitet. ${ }^{24}$ Nach Jaspers ,ist die Konsequenz des unbedingten religiösen Handelns, daß allein in ihm das eigentliche Sein ergriffen wird.“25 Wenn das religiöse Handeln absolut ist, dann kann nichts vor ihm bestehen. Auf nichts anderes kann es ankommen. Abgesehen von jenen (pseudo-)religiösen Formen (Magie, Aberglauben, Mythologien der Völker usw.), in denen die Beziehung zur Transzendenz innerweltlichen Zwecken untergeordnet ist, kann die engste Verknüpfung zwischen Religion und Weltverneinung konstatiert werden. Üben in den obigen innerweltlichen (pseudo-) religiösen Formen der religiösen Handlung Opfer und asketische Handlungen als einzelne Leistungen Zwänge auf die Gottheit aus, um

21 Jaspers, Philosophie, Bd. I, S. $81 \mathrm{f}$.

22 Karl Jaspers, Der philosophische Glaube, München 1948, S. 32.

23 Jaspers, Der philosophische Glaube, S. 33.

24 Jaspers, Philosophie, Bd. II, S. 292-336. Im Folgenden fasse ich den Unterabschnitt „Religiöse Weltverneinung“ zusammen, siehe S. 318-320.

25 Jaspers, Philosophie, Bd. II, S. 318. 
immanente Zwecke zu erfüllen, so wird erst in den Religionen die Erscheinung der totalen Askese wirklich.

In ihrer reinen Form ist diese Verneinung ein negativer Entschluss, d. h. ein negativer existenzialer Akt, in dem eine negative Existenzform konstituiert wird. Diese weltverneinende Existenz identifiziert sich weder mit einer Objektivität der Welt noch mit einer Subjektivität ihres eigenen Daseins; sie tritt in keine unbedingte Kommunikation. Weltlos und kommunikationslos will sie, isoliert auf die Transzendenz bezogen, für diese die Totalität der Welt negieren, auch wenn diese Haltung in der Welt notwendigerweise von ambivalentem Charakter ist: In der Welt die Welt verlassen, ist objektiv unmöglich und so realisiert das religiös weltverneinende Individuum eine widersprüchliche, unglückliche Existenzform. Diese Seinsform ist ein aufreibendes negatives Dasein der Existenz als verlorene Einsamkeit mit einer Gottheit, die sich neben der Welt vollzieht, in der die Existenz doch bleiben muss. Doch ist diese Existenzform für das Dasein unentbehrlich: Sie lenkt nämlich den Blick auf die Fragwürdigkeit allen Daseins. Wenn sie sich selbst als das Wahre objektiviert, wird sie ein „Irrlicht“26, wenn sie aber die ruhige Harmonie eines Weltdaseins überhaupt stört, dann wird sie die Wahrheit. „Dass in aller Zeit Menschen die Weltlosigkeit gesucht $\mathrm{zu}$ haben schienen, ist ein nie aufhörendes memento, gesprochen an das Dasein möglicher Existenz in der Welt, wenn es selbstgerecht werden will in seinem Glück.“27

Der negative Entschluss ist bei Jaspers ein Analogon zum Selbstmord. Aus dem bereits Gesagten folgt aber, dass keine mögliche Existenz wagen darf, diese existentiale Form der negativen Weltbeziehung für schlechthin unwahr zu erklären. „An der Grenze dessen, was Menschen möglich ist, stehen diese Heroen des Negativen, und bringen sich zum Opfer. In ihrer furchtbaren Einsamkeit zeigen sie, was, einmal gesehen, in der Welt unverlierbar eine Wirklichkeit bedeutet, die jede selbstzufriedene Ruhe im Keim erstickt." ${ }^{28}$ Der negative Entschluss ist also zwingend widersprüchlich, denn er ist nicht lediglich die Leugnung eines konkreten innerweltlichen Zusammenhanges, sondern vielmehr eine existentielle, vom Anspruch eines unmittelbaren, außerweltlichen Verhältnisses motivierte, radikale Negation der Weltbezogenheit des menschlichen Seins. Diese existentielle Negation wird nach Jaspers gerade und allein dadurch zu einem heroischen Akt, dass das Individuum zu einer verwirrenden und beunruhigenden Ausnahme wird, das auf die grundsätzliche Fragwürdigkeit der Existenz aufmerksam macht.

26 Jaspers, Philosophie, Bd. II, S. 319.

27 Ebd.

28 Jaspers, Philosophie, Bd. II, S. 320. 


\section{Die Idee des religiösen Akosmismus in Jaspers' Kierkegaard-Deutung}

Auch wenn der Ausdruck des religiösen Akosmismus in Jaspers' publizierten Schriften nicht vorkommt, scheint mir, dass sich der Grund der immanenten Ambivalenz seines Verhältnisses zu Kierkegaard präzise an diesem Begriff erklären lässt. Die inhaltliche Entsprechung des religiösen Akosmismus findet sich in den von Jaspers in diesem Kontext verwendeten Ausdrücken „religiöse Weltverneinung“ bzw. die „Weltlosigkeit des religiösen Subjektes“. Es ist terminologisch bemerkenswert, dass die Formulierung der „akosmischen Beziehung zu Gott“, die die negative Seinsposition des Kierkegaardschen „Einzelnen“ (Enkelte) bezeichnet und eine stark kritische Funktion besitzt, auch in der KierkegaardAuslegung des Jaspers-Zeitgenossen Martin Buber (1878-1965) auftaucht. ${ }^{29}$ Durch diesen Begriff bezeichnet Buber bei Kierkegaard eine die Welt ausschließende, rein negative Relationalität. „Das einzige holistische Verhältnis des Einzelnen zu einem Anderen ist seine Beziehung zum Absoluten, d.h. zu Gott. Diese verabsolutierte Beziehung wird von Buber nicht nur als ausschließlich sondern auch als ausschließend klassifiziert, da sie alle anderen Beziehungen 'ins Reich der Unwesenheit' verbannt. “30 Man denke nur an Sätze wie die folgenden aus Kierkegaards Schrift Der Einzelne: „[E]in jeder soll mit 'den anderen' nur vorsichtig sich einlassen, wesentlich allein mit Gott und sich selber reden.“31 „Als 'der Einzelne’ ist er allein, allein in der ganzen Welt, allein - Gott gegenüber.“32 Behauptungen wie jene führen Buber zu der Annahme, bei Kierkegaard würden die Sphären der Intersubjektivität in Folge der Exklusivität des Gottesverhältnisses entwertet, ja

29 Martin Buber, Die Frage an den Einzelnen, Berlin 1936; ders., Die Schriften über das dialogische Prinzip, Heidelberg 1954. Hiermit möchte ich Peter Šajda meinen Dank aussprechen, dass er meine Aufmerksamkeit auf diesen rezeptionsgeschichtlichen Problemkomplex gelenkt hat; ebenso dafür, dass er mir das unveröffentlichte Manuskript seines Vortrages „Das Problem des religiösen Akosmismus: Bubers Kritik an Kierkegaard“ zur Verfügung stellte. Zu Martin Bubers Kierkegaard-Rezeption siehe seine gründliche Studie „’No-One Can So Refute Kierkegaard as Kierkegaard Himself'“, in Kierkegaard and Existentialism, hg. von Jon Stewart, Aldershot 2011 (Kierkegaard Research: Sources, Reception and Resources, Bd. 9), S. 33-61. Ferner siehe: Peter Šajda, „A Jewish, a Catholic and a Neo-Marxist Critique of Kierkegaard's Philosophy of Religion“, in Kierkegaard Studies Yearbook, 2012, S. 303-321; ders., Buberov spor s Kierkegaardom. O vzt'ahu náboženstva $k$ etike a politike, Bratislava 2013.

30 Peter Šajda, „Das Problem des religiösen Akosmismus: Bubers Kritik an Kierkegaard“, unveröffentlichtes Manuskript.

31 SKS 16, 86 / GWS, 99.

32 SKS 16, 103 / GWS, 117. 
abgeschafft, das Individuum isoliert und in sich eingeschlossen. Der Begriff Akosmismus bezeichnet folglich in dieser Lesart nicht die Negation der ontologischen Realität der Welt, ${ }^{33}$ sondern eine religiös motivierte, grundsätzlich negative Einstellung zur Welt. In der religiösen Weltverneinung wird nicht die Substanz, sondern die existentielle Relevanz der Welt negiert: Für den Glaubenden ist alles, was außerhalb seines Verhältnisses zum Absoluten steht, wesentlich nichtig.

Im Folgenden zeige ich in historisch-systematischer Perspektive, welche Rolle die Problematik des Akosmismus im Kontext der Kierkegaard-Rezeption von Karl Jaspers eigentlich spielt. Es ist zunächst auffällig, dass Jaspers das Problem der Weltverneinung bei Kierkegaard de facto früher als Buber behandelt, jedoch haben seine diesbezüglichen, frühen Reflexionen keinen wirklich kritischen Charakter, sondern sind eher neutraler, deskriptiver Art. Jaspers diagnostiziert lediglich die Negativität des Kierkegaardschen Denkens, doch ohne ihn deswegen zu kritisieren. In seinen späteren Schriften, besonders in Der philosophische Glaube angesichts der Offenbarung (1962), wird die Verwendung dieses Begriffes allerdings kritisch, und die Destruktivität der Glaubenskonzeption Kierkegaards wird breit analysiert und letztlich entschieden abgewiesen. Der Grund für diese Akzentverschiebung liegt meines Erachtens weniger in einem etwaigen konzeptionellen Bruch, der für Jaspers’ über Jahrzehnte währende Kierkegaard-Rezeption

33 Obwohl Kierkegaard den Begriff Akosmismus nicht oft verwendet, hat der Ausdruck in einigen Passagen eine besondere Relevanz. Merkwürdigerweise wird die Position des Christentums jener des ontologisch aufgefassten Akosmismus bereits in den Journalpassagen über Martensens spekulative Dogmatik gegenübergestellt: „In der Bestimmung von Gottes Verhältnis zur Welt negiert das Xstt. auf der einen Seite den Akosmismus, indem das Xstt. die Welt und die Endlichkeit nicht als Schein betrachtet, sondern der Kreatürlichkeit eine relative Wirklichkeit und Selbstständigkeit außerhalb Gottes zuschreibt; auf der anderen Seite wird der abstrakte Dualismus negiert, indem die Schöpfung nicht bloß aus Gott als ihrer absoluten Ursache ist, sondern zugleich bei Gott besteht.“ SKS 18, 382, KK:11 / DSKE 2, 392. (Bezüglich dieser Passage ist unbedingt darauf hinzuweisen, dass das Journal KK:11 das Exzerpt eines Kollegabonnements ist, das Kierkegaard als Ersatz für fehlende Lehrbücher diente. Siehe dazu SKS K18, 484-488 / DSKE 2, 669-673.) Nicht weniger relevant ist Kierkegaards Verwendung des Begriffs in der Dissertation in Bezug auf Fichte: „Indem Fichte dergestalt das Ich verunendlichte, machte er einen Idealismus geltend, in Bezug auf den alle Wirklichkeit verblich, einen Akosmismus, in Bezug auf den sein Idealismus Wirklichkeit wurde, obwohl er Doketismus war." SKS 1, 309 / BI 278. Schließlich spielt Johannes Climacus in der Nachschrift ironisch auf ein mögliches spekulatives Missverständnis seiner Konzeption über die ethische Wirklichkeit des Einzelnen als einer Form des Akosmismus an: „Dem Denken die Suprematie über alles andere zu geben, ist Gnostizismus, die ethische Wirklichkeit des Subjekts zur einzigen Wirklichkeit zu machen, könnte Akosmismus zu sein scheinen.“ SKS 7, 312 / AUN2, 45. 
alles andere als typisch wäre ${ }^{34}$; vielmehr handelt es sich um eine thematische Transposition innerhalb seines Interesses, da er sich in seiner späteren Periode, besonders nach seiner Emigration in die Schweiz, auf das Verhältnis des philosophischen und christlichen Glaubens konzentrierte - nicht zuletzt unter dem Einfluss der unmittelbaren Erfahrungen, die er mit dem Denken seines Basler Kollegen Karl Barths gemacht hatte, dessen frühe, dialektische Theologie bekanntlich stark unter dem Einfluss Kierkegaards stand. ${ }^{35}$

Aus der Psychologie der Weltanschauungen geht hervor, dass Jaspers bereits in seinem frühesten philosophischen Werk den akosmischen Charakter des Kierkegaardschen Denkens betont. Er analysiert Kierkegaards Konzeption in Der Krankheit zum Tode im Zusammenhange mit der existentiellen Grenzsituationen und der psychologischen Interpretation der Sünde und stellt fest: „Kierkegaard zeigt die Situation, verneint die Verendlichungen, sagt nichts von der Art des weltanschaulichen Erlebens selbst. Er lässt nur merken, dass diese Kräfte ganz außerweltlich sind: einsam mit Gott. Er gibt keine Impulse zur Weltgestaltung. Das Ethische ist Problem der Stellungnahme zu sich selbst, die Zwecke werden alle direkt auf das Absolute abgestellt, nicht durch Welthandeln hindurch. Es ist eine reflexive, einsame Religiösität des Denkens.“36

Gegen Kierkegaards solipsistischen, akosmischen Glauben macht Jaspers später, im dritten Band der Philosophie (1931), auch inhaltlich klar, dass der einzige Bereich des Verhältnisses zur Transzendenz die Einheit von Welt und Geschichte ist. Es gibt also keine direkte, unmittelbare, weltlose Beziehung zum Absoluten. „Weltlose Liebe ist Liebe zu nichts als grundlose Seligkeit. Liebe zur Transzendenz ist nur als liebende Weltverklärung wirklich. “37 In den Groningen-Vorlesungen schließlich (1935), in denen Jaspers seine umfangreichste Kierkegaard-Interpretation präsentiert, wird erneut die Negativität des religiösen Weltverhältnisses hervorgehoben. „Alles Bestehende wird gleichsam verzehrt in einer schwindelerregenden Bewegung durch die Saugkraft: bei Kierkegaard eines außerweltlichen Christentums, das wie das Nichts ist und nur in Verneinung (dem Absurden, dem Märtyrersein) und im negativen Entschluss sich zeigt. “38 Kierkegaard macht einen

34 Dies wird auch von Ehrlich bestätigt. Siehe dazu: Leonard Ehrlich, „Jaspers Reading Kierkegaard: An Instance of the Double Helix“, In Karl Jaspers on Philosophy of History and History of Philosophy, hg. von Joseph W. Kotersky und Raymond J. Langley, Amherst, New York 2003, S. 239.

35 Zu Jaspers' ambivalenter Einstellung zu Barth siehe: Hans Saner, Karl Jaspers in Selbstzeugnissen und Bilddokumenten, Hamburg 1970, S. $148 \mathrm{f}$.

36 Jaspers, Psychologie der Weltanschauungen, S. 247.

37 Jaspers, Philosophie, Bd. III, S. 167.

38 Jaspers, Vernunft und Existenz, S. 6. 
Sprung „zum Christentum, aufgefasst als absurde Paradoxie, als der negative Entschluss des völligen Weltverzichts, und als notwendiges Märtyrersein. “ ${ }^{39}$ Hier sei auch erwähnt, dass Jaspers als Verwirklichungsformen der Kierkegaardschen Weltverneinung meist jene beiden Momente der Absurdität und des Martyriums nennt.

In seinen Basler Vorlesungen bestimmt er Gott wie folgt als philosophischen Glaubensgehalt: „Transzendenz über aller Welt oder vor aller Welt heißt Gott.“40 Mit dieser Feststellung wären alle Pseudonyme Kierkegaards ohne Vorbehalt einverstanden. Die entscheidende Differenz zwischen den beiden Autoren liegt nicht in der Problematik des absoluten Andersseins der Transzendenz, woraus ihre Unbegreiflichkeit für das Denken folgt, sondern in der Frage nach der Möglichkeit des Verhältnisses des Existierenden zur Transzendenz. Während nämlich die Weltlichkeit der Existenz bei Jaspers eine conditio sine qua non für das Transzendenzverhältnis bildet, ist dieselbe Weltbezogenheit für Kierkegaard eben ein Hindernis für jenes Verhältnis. Darauf gründet sich die Negativität der Entscheidung für den absurden Glauben, die als ein praktisches Prinzip von Jaspers folgendermaßen charakterisiert wird: Es ist „kein Beruf zu ergreifen, keine Ehe zu schließen“, denn „allein das Märtyrersein ist eigentliches Christsein“ “41.

Das Problem der religiösen Weltverneinung spielt eine zentrale Rolle auch in Jaspers' Spätwerk Der philosophische Glaube angesichts der Offenbarung (1962), in dem die kritische Haltung ganz offenkundig dominiert. In diesem Werk wird Kierkegaard eingehend behandelt, ${ }^{42}$ die Interpretation konzentriert sich aber bezeichnenderweise auf einen kritischen Punkt, nämlich auf Kierkegaards späten, scharfen Konflikt mit der dänischen Kirche. Jaspers misst diesem Konflikt eine entscheidende Bedeutung bei und nimmt an, dass diese Kollision notwendigerweise aus Kierkegaards Konzeption der Absurdität des Glaubens folgt. Es wäre s.E. trotzdem ein gänzliches Missverständnis zu behaupten, Kierkegaard kritisiere die bestehende Christenheit vom Standpunkt des authentischen Christentums aus. Was Kierkegaard eigentlich wollte, das ist nach Jaspers nichts anderes als die Redlichkeit (Redelighed). Dieser „Wille zur Redlichkeit“ zeigt sich vielleicht am klarsten in einem späten Zeitungsartikel mit dem Titel: „Was will ich?“43, dessen erster Satz bereits lautet: „Ganz einfach: ich will Redlichkeit.“ In dieser Schrift

39 Jaspers, Vernunft und Existenz, S. 15.

40 Jaspers, Der philosophische Glaube, S. 29.

41 Jaspers, Der philosophische Glaube, S. 128.

42 Siehe: Karl Jaspers, Der philosophische Glaube angesichts der Offenbarung, München 1962, S. $513-525$.

43 Søren Kierkegaard, „Hvad jeg vil?“, in Fædrelandet, Nr. 77, 31. März 1955, S. 317; vgl. SKS 14, $179-181 / A, 48-52$. 
charakterisiert der Autor sich selbst ziemlich ungewöhnlich: „Auf keine Weise, ich bin weder Milde noch Strenge - ich bin: Menschliche Redlichkeit." ${ }^{44}$ Die Bedeutung dieser Feststellung lässt sich schwerlich überschätzen. In diesem Anspruch auf Redlichkeit hallt nämlich der Grundton der Rhetorik jener philosophischen Strömungen wider, die in der Auflösungszeit der Systeme des Deutschen Idealismus aufkamen. Während Schopenhauer die Deutschen Idealisten als Sophisten und unredliche Scharlatane bezeichnet ${ }^{45}$ und Nietzsche den Anspruch der intellektuellen Redlichkeit nicht nur aufstellt, sondern durch die Umwertung aller Werte auch verwirklicht, enthüllt Kierkegaard die Unchristlichkeit der bestehenden Christenheit mit einer schonungslosen Radikalität, eben auf dem Grunde des Imperativs der Redlichkeit, mit dem er sich selbst rhetorisch identifiziert. Die Wahrheit Kierkegaards liegt nach Jaspers genau in der Unbedingtheit seines Willens zur Redlichkeit, der nicht weniger radikal ist als Nietzsches späte bekannte Selbstbezeichnung: „Ich bin kein Mensch, ich bin Dynamit.“46

Zugleich formuliert Jaspers genau an dieser Stelle die schärfste und grundlegendste Kritik an Kierkegaard: „Wer sich entschieden hat,“ schreibt er, „darf sagen: Ich halte den Weg, zu dem Kierkegaard durch die Pseudonyme Johannes Climacus und Anticlimacus verleitet, für einen Irrweg: zum Glauben kraft des Absurden mit einem verführerisch erdachten genialen Kategoriengebäude, mit seiner Konstruktion der Nachfolge Christi im eindeutigen Sinn totaler Weltverneinung. “47 Eine Aufzeichnung aus Jaspers' handschriftlichem Nachlass macht die vernichtende Kritik an Kierkegaards Glaubensauffassung deutlich: „Gegen die Konstruktion des Johannes Climacus und gegen den Glauben Kierkegaards: / Fiktion, - daher die 'Schuld' des Nein - / der 'Verrat' am Menschen, am Geliebten, an der Welt / das faktische Weglaufen, wie das des Selbstmörders?“48 Im selben Text wird auch das Paradox des Glaubens als eine „weltlose Transzendenz der isolierten Seele mit Gott“49 gedeutet.

Da der religiöse Akosmismus ebenfalls die Negation der interpersonalen Verhältnisse einschließt, folgt schließlich aus diesem Konzept die Liquidation der religiöse Gemeinde: „Wenn Kierkegaard Recht hätte, so wäre es das Ende des

44 SKS 14, 179 / A, 48.

45 Siehe Arthur Schopenhauer, Die Welt als Wille und Vorstellung, Bd. I, 2. Aufl., Leipzig 1844, S. XIX.

46 Friedrich Nietzsche, Ecce homo. Wie man wird, was man ist, in Nachgelassene Werke von Friedrich Nietzsche, in Nietzsches Werke, Abt. II, Bd. 15, Leipzig 1922, S. 116.

47 Jaspers, Der philosophische Glaube angesichts der Offenbarung, S. 516.

48 Karl Jaspers, Die großen Philosophen, hg. von Hans Saner, Bd. II., München-Zürich 1981, S. 825.

49 Jaspers, Die großen Philosophen, Bd. II., S. 817. 
Christentums in der Welt. Wenn der christliche Glaube das ist, als was Kierkegaard ihn konstruiert hat, dann kann ihn vielleicht niemand mehr glauben, wie denn Kierkegaard für seine Person diesen Glauben auch nicht in Anspruch genommen, ihn aber leidenschaftlich begehrt hat. “50 Diese immanente Negativität der existenzdialektischen Christentumsauffassung nach Kierkegaard führt de facto zum Ende des Christentums: „Ein mit der theologischen Konstruktion Kierkegaards in seiner Unverständlichkeit verstandener, zur Absurdität gezwungener christlicher Glaube, der sich damit in einer modernen Welt gleichsam rechtfertigt, könnte ebenso wie Kierkegaards Entlarvung der Kirchlichkeit das Ende dieses Christentums und dieser Kirche bedeuten. ${ }^{\text {"51 }}$ Die Jasperssche Kritik an Kierkegaards antagonistischer Verhältnisbestimmung des Glaubenden zur Welt stützt sich zusehends auf eine reductio ad absurdum: Wenn nämlich Kierkegaard Recht hätte, dann könnte das Christentum gar nicht bestehen. Da aber das Christentum tatsächlich besteht, wird Kierkegaards Konzeption ipso facto fragwürdig. Von Kierkegaards Standpunkt aus scheint es aber, dass Jaspers' Konklusion aus seinen Prämissen gar nicht folgt - was nämlich in der Tat besteht, ist nur Schein, oder eine Parodie, ${ }^{52}$ eine Verfälschung und ein Verrat des Christentums. ${ }^{53}$ Allerdings ist dieser Widerspruch nur ein scheinbarer: Während nämlich Jaspers von der Faktizität der Christentums ausgeht, behauptet Kierkegaard die christliche Idealität, das Christentum (Christendom), von dem er die bestehende Christenheit (Christenhed) terminologisch trennt. ${ }^{54}$

Jaspers betrachtet also das empirisch-historische Bestehen des Christentums als eine evidente Tatsache und kritisiert die religiös motivierte Weltverneinung Kierkegaards in der Spätperiode seines Denkens scharf. Obwohl dessen Weltverneinung christlicher Herkunft ist, ist sie paradoxerweise eine Negation der Religion selbst und gründet sich eigentlich auf die Konzeption eines religionslosen Gottesglaubens. Doch folgert Jaspers nirgends, dass Kierkegaards Denken im Grunde genommen falsch oder irreführend wäre. Seiner Meinung nach ist nämlich Kierkegaard eine „Ausnahme“ ${ }^{55}$ ein „großer Erwecker“, ${ }^{56}$ ein „Heroe des

50 Jaspers, Der philosophische Glaube angesichts der Offenbarung, S. 517.

51 Jaspers, Der philosophische Glaube angesichts der Offenbarung, S. 518.

52 Vgl. z.B. SKS 7, 338 / AUN2, 75.

$53 \mathrm{Zu}$ Kierkegaards Behauptung, dass das Christentum nicht mehr da ist, siehe seine späten, kirchenpolemischen Schriften, z. B.: SKS 216, 237 ff. / A 167, $184 \mathrm{ff}$.

54 Siehe dazu SKS 13, 235f. / A $182 \mathrm{ff}$.

55 Jaspers, „Kierkegaard. Zu seinem 100. Todestag“, in ders., Aneignung und Polemik, S. 316. 56 Siehe dazu: István Czakó, „Karl Jaspers: A Great Awakener’s Way to Philosophy of Existence“, in Kierkegaard and Existentialism, hg. von Jon Stewart, Aldershot 2011 (Kierkegaard Research: Sources, Reception and Resources, Bd. 9), S. 155-197. 
Negativen“, ${ }^{57}$ ein „Prophet der indirekten Mitteilung“558 und als solcher ist er für das existentielle Selbstverständnis schlechterdings unentbehrlich; gleichwohl, ihm tatsächlich zu folgen, sei ebenso unnötig wie unmöglich. Es ist freilich kaum überraschend, dass Jaspers an der denkerischen Leistung Kierkegaards nicht die weltverneinende, akosmische Christentumsauffassung schätzt: „Die Größe Kierkegaards liegt kaum in seiner Interpretation des christlichen Glaubens, der von ihm vielmehr in eine Form gebracht wird, die als Selbstvernichtung dieses Glaubens anmutet. Es ist eine raffinierte Dialektik der unaufgelösten Widersprüche, die in diesen selber die Wahrheit der Wirklichkeit Gottes entdeckt. “59

\section{Schlussbemerkungen zu Jaspers' Kritik an Kierkegaard}

Abschließend möchte ich noch einige kurze Bemerkungen zu Jaspers’ Kritik und Methodologie im Bereich seiner Kierkegaard-Rezeption hinzufügen. Zunächst ist festzustellen, dass Jaspers mit Kierkegaards Euvre imponierend gründlich und umfassend vertraut war, obwohl er das Gesamtwerk aus sprachlichen Gründen nicht im Original und also nicht restlos rezipieren konnte. Es scheint mir aber, dass er Kierkegaard ebenso „rückwärts“ gelesen hat, wie einst vermutlich Hegel von dem jungen Kierkegaard aufgenommen wurde. Anzeichen hierfür bietet nicht nur die Tatsache, dass sich Jaspers mit den Texten des späten Kirchenkampfs bereits früh vertraut machte, sondern auch, dass er diesen Texten eine entscheidende Bedeutung für das Gesamtverständnis Kierkegaards beimessen konnte. Kierkegaards späte, polemische Schriften bilden praktisch den wichtigsten Schlüssel für Jaspers' retrospektive Kierkegaard-Lektüre. Dieses hermeneutische Prinzip ist seiner Meinung nach keineswegs willkürlich, denn Kierkegaards Konflikt mit dem dänischen Staatschristentum war demnach eine unmittelbare und notwendige Folge seiner in den pseudonymen Schriften ausgebildeten Glaubenskonzeption. ${ }^{60}$ Zweifellos üben die späten, polemischen Schriften eine bedeutende Wirkung auf die frühe deutsche Kierkegaard-Rezeption aus. Ebenso unstrittig ist die Möglichkeit einer konsistenten Rekonstruktion der Grundkonzeption dieser Schriften auf der Basis der Begriffe „Akosmismus“ und „Weltverneinung“: Das authentische Christsein konstituiert sich im negativen Entschluss und kulminiert im Martyrium.

57 Jaspers, Philosophie, Bd. II, S. 320.

58 Jaspers, Psychologie der Weltanschauungen, S. 375.

59 Jaspers, Die großen Philosophen, Bd. II, S. 845.

60 Jaspers, Der philosophische Glaube angesichts der Offenbarung, S. 518. 
Darin jedoch eine Evidenz für die Anwendbarkeit dieser Hermeneutik auf das ganze Euvre zu sehen, scheint mir zweifelhaft. Jaspers' Verfahren, die Glaubenskonzeption der späten, kirchenpolemischen Schriften auf das Gesamtwerk hermeneutisch zu extrapolieren, bleibt damit zumindest fragwürdig.

Zur Begründung soll hier ein exemplarischer Hinweis darauf genügen, dass sich unter anderem die Schwerpunkte der Glaubensauffassung Kierkegaards auffallend verlagert haben: Während die Glaubenskonzeption der frühen Schriften auf die Kategorie der verborgenen Innerlichkeit (Inderlighed) aufgebaut ist, wird der Glaube in den späten, nach der Unwissenschaftlichen Nachschrift veröffentlichten Schriften eher im Horizont des Konflikts mit der bestehenden Ordnung und mit der Welt interpretiert, wodurch die Kategorie des Martyriums in den Vordergrund gerät, das keineswegs, verborgen' ist. In Furcht und Zittern vollzieht Abraham die Bewegung der unendlichen Resignation ${ }^{61}$ und isoliert sich durch seine negative Entscheidung - doch er ist kein Märtyrer, sein Glaube keine Weltverneinung, sondern eine neue Unmittelbarkeit, eine Rückkehr zur Endlichkeit, eine Wiederholung. In der Nachschrift wird die mittelalterliche Klosterbewegung deshalb kritisiert, weil sie das absolute, pathetische Verhältnis zum Absoluten auf eine inadäquate, weil objektive Weise auszudrücken versucht. ${ }^{62}$ Dieses Verhältnis ist für Climacus keine akosmische Negation der objektiven, äußerlichen Verhältnisse, sondern ein Inkognito, in dem die endlichen Ziele der Welt zwar relativiert, aber nicht gänzlich negiert werden.

Mir scheint daher, dass Jaspers zwar nicht unbegründet, doch zweifellos einseitig und vereinfachend das Ganze der Glaubenskonzeption Kierkegaards für weltverneinend erklärt. Ebenso vereinfachend ist Jaspers' Interpretation des Absurden als einer Form des negativen Entschlusses und der Weltverneinung. Das Absurde ist bei Climacus keine bloße Negation der Vernunft. Dies zeigt nicht nur der Abschnitt „Das Dialektische“ in der Nachschrift, in dem Climacus zwischen absurd und unsinnig scharf unterscheidet, ${ }^{63}$ sondern auch eine Passage des Notizbuches 13, in der der Glaube - in Übereinstimmung mit Leibniz - als ein Verhältnis supra rationem aufgefasst wird. ${ }^{64}$ Für die Verfasserperspektive eines Außenstehenden erscheint der Glaube noch als Paradox und Absurdes, während in den späteren, in einer eminent christlichen Perspektive konzipierten Texten diese Problematik beinahe völlig in den Hintergrund rückt. Eine Aufzeichnung über Magnus Eiríksson um 1850 stellt sogar fest: „Indem der Glaubende glaubt, ist

61 Siehe SKS 4, 132 / FZ, 35.

62 Siehe dazu SKS 7, 368-382 / AUN2, 111-126.

63 SKS 7, 516 / AUN2, 280.

64 SKS 19, 390f., Not13:23 / T 1, 350. 
das Absurde nicht das Absurde - der Glaube verwandelt es. “65 Zwar wird Jaspers” Kierkegaard-Interpretation durch die obigen Passagen nicht regelrecht widerlegt; zumindest aber kommt durch sie die Selektivität und Einseitigkeit seiner Konzeption eindeutig zum Vorschein.

In seinem Vortrag „Kierkegaard heute“ erklärt Jaspers pathetisch und nicht ohne Grund: „Wer Kierkegaard eigentlich war, was er sagen wollte, das weiß, meine ich, niemand."66 Trotzdem zeigt die Rezeptionsgeschichte mannigfach, dass viele der Auffassung waren und sind, diese beiden Fragen restlos beantworten zu können. Eine der wichtigsten Aufgaben der heutigen KierkegaardForschung liegt m. E. darin, die herkömmlichen philosophiegeschichtlichen Mythen zu revidieren und einem hermeneutisch sachgemäßeren Verständnis Kierkegaards den Weg zu ebnen.

65 Pap. X-6 B 79, 86 / T 5, 385. Zu Kierkegaards Verhältnis zu Magnus Eiríksson siehe: Gerhard Schreiber, „Magnus Eiríksson: An Opponent of Martensen and an Unwelcome Ally of Kierkegaard“, in Kierkegaard and His Danish Contemporaries, Tome II, Theology, hg. von Jon Stewart, Aldershot 2009 (Kierkegaard Research: Sources, Reception and Resources, Bd. 7), S. 49-94. 66 Jaspers, Aneignung und Polemik, S. 322. 